\title{
Er:YAG single-crystal fiber laser in Q-switched operation
}

\author{
Igor Martial, ${ }^{1,2, *}$ Julien Didierjean, ${ }^{2}$ François Balembois, ${ }^{1}$ and Patrick Georges ${ }^{1}$ \\ ${ }^{1}$ Laboratoire Charles Fabry de l'Institut d'Optique, CNRS, Université Paris-Sud, Campus Polytechnique, RD 128, 91127 Palaiseau Cedex, \\ France \\ ${ }^{2}$ Fibercryst SAS, La Doua-Bâtiment l'Atrium, Boulevard Latarjet, F- 69616 Villeurbanne Cedex, France \\ "Corresponding author: igor.martial@institutoptique.fr
}

\begin{abstract}
We describe an efficient Q-switched laser emission from a directly grown $\mathrm{Er}^{3+}: \mathrm{YAG}$ single-crystal fiber resonantly pumped by a laser diode in an off-axis configuration. The laser produces $2 \mathrm{~mJ}, 38 \mathrm{~ns}$ pulses at $1 \mathrm{kHz}$.

OCIS codes: (140.3580) Lasers, solid-state, (140.3480) Lasers, diode-pumped, (140.3540) Lasers, Q-switched, (140.3380)

Laser materials, (140.3500) Lasers, erbium.
\end{abstract}

\section{Introduction}

The yttrium aluminium garnet (YAG) crystal doped with $\mathrm{Er}^{3+}$ is one of the most attractive materials for the development of multi-watt compact eye safe laser sources around $1.6 \mu \mathrm{m}$. Such sources are required for many applications like remote sensing, free-space communications, range detection, and designation. The optical transition between the ${ }^{4} \mathrm{I}_{15 / 2}-{ }^{4} \mathrm{I}_{13 / 2}$ manifolds includes the possibility for in-band pumping (between 1.45 and 1.53 $\mu \mathrm{m})$. This minimizes the heat generated by the pumping process and hence limits the thermally induced birefringence and the thermal lens. Er:YAG lasers can be resonantly pumped using erbium-doped fiber lasers (EDFL) [1-4]. The excellent beam quality of EDFL allows good spatial overlap of the pumped volume with the laser mode and efficient pump absorption due to the narrow linewidth. High power and good optical efficiency have been achieved using this EDFL pumping [3]. However, this pumping approach adds complexity, weight and volume to the laser system. Additionally, the overall optical to optical efficiency with EDFL pumping is typically only about $28 \%$ due to the number of stages [3].

Direct pumping using high power laser diodes can be a solution to improve the overall efficiency of the system [5-8]. However, such direct pumping is complicated because of the low brightness of laser diodes. For this purpose, the use of long and thin rods (single-crystal fiber), confining the pump beam close to the signal beam, represents one way to manage the poor beam quality of pump laser diodes and to achieve high pump intensities along the whole laser medium [9]. However, these rods are difficult to manufacture from standard Czochralski-grown boules: after cutting and complex polishing of the cylinder, it is difficult to reduce the diameter below $1 \mathrm{~mm}$ for a few centimeter long sample. The micro-pulling-down technique $(\mu \mathrm{PD})$ provides a solution to this problem as laser rods can be elaborated directly, without any additional polishing of the cylinder [10]. The diameter can be easily reduced to a few hundreds of microns and typical crystal lengths can be up to $80 \mathrm{~cm}$. In a previous work [11], we demonstrate the growth of laser quality Er:YAG single-crystal fiber by the micro-pulling-down technique and the first laser ever achieved with such gain medium.

In this work, we improve the setup by using the all potential of single-crystal fiber in an off-axis pumping configuration. We report Q-switched operation of a directly grown Er:YAG single-crystal fiber for the first time to our knowledge.

\section{Experiment}

The experimental setup is shown in Fig. 1 and consists of an off-axis end-pumped actively cooled $0.5 \% \mathrm{Er}^{3+}: \mathrm{YAG}$ single-crystal fiber which has a diameter of $800 \mu \mathrm{m}$, a length of $60 \mathrm{~mm}$ and anti-reflection coatings on both ends. We use a bi-concave cavity. The first concave mirror is highly reflective at $1645 \mathrm{~nm}$ and the second one has a transmission of $30 \%$. The pump light is provided by a fiber-coupled laser diode, with a $400 \mu \mathrm{m}$ core diameter and a numerical aperture of NA $=0.22$, delivering up to $80 \mathrm{~W}$ at $1532 \mathrm{~nm}(\sim 1 \mathrm{~nm}$ FWHM). The fiber is imaged into the crystal by a 1:1 telescopes consisting of two AR coated lenses L1 and L2 with the focal lengths of $50 \mathrm{~mm}$. In order to maximize the output power, the focus point is put slightly inside the single-crystal fiber, close to the pumped face. It corresponds to a $400 \mu \mathrm{m}$ diameter beam. The pump beam is then guided inside the single-crystal fiber by total internal reflections. 


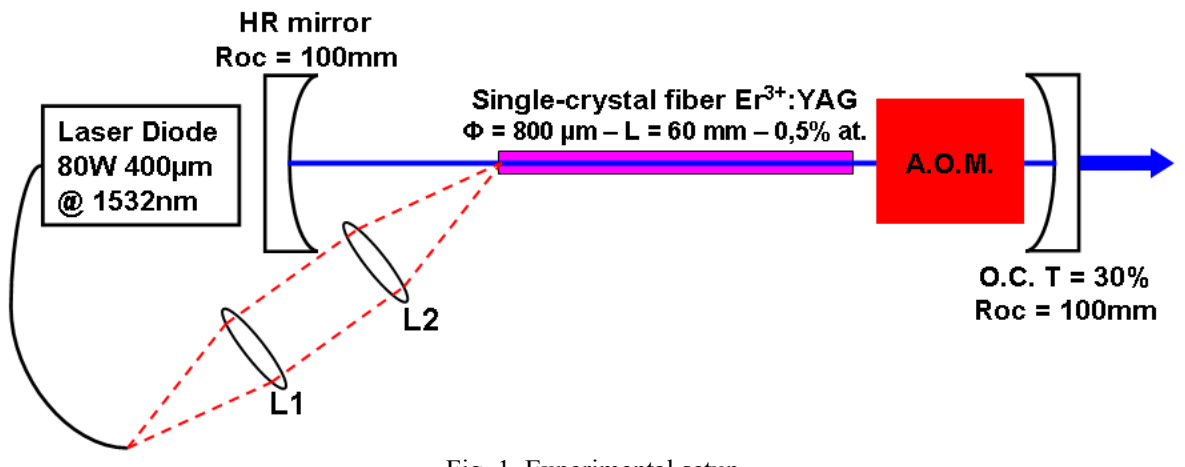

Fig. 1. Experimental setup

This off-axis configuration enables to keep a good overlap between the pump and signal beams, without the need of a dichroic mirror to combine both. This offers an important advantage because of the complex manufacturing and low damage threshold of dichroic mirrors designed for such a small difference between the signal and pump wavelength.

\section{Results}

We first tested the laser in CW operation. With a transmission of $20 \%$ for the output coupler, the measured absorbed pump laser threshold was $15.8 \mathrm{~W}$ and the maximum output power at $1645 \mathrm{~nm}$ was $7.3 \mathrm{~W}$ for $58 \mathrm{~W}$ of absorbed pump power, leading to a slope efficiency of $18 \%$. Fig. 2 reports the measured output power versus the absorbed pump power.

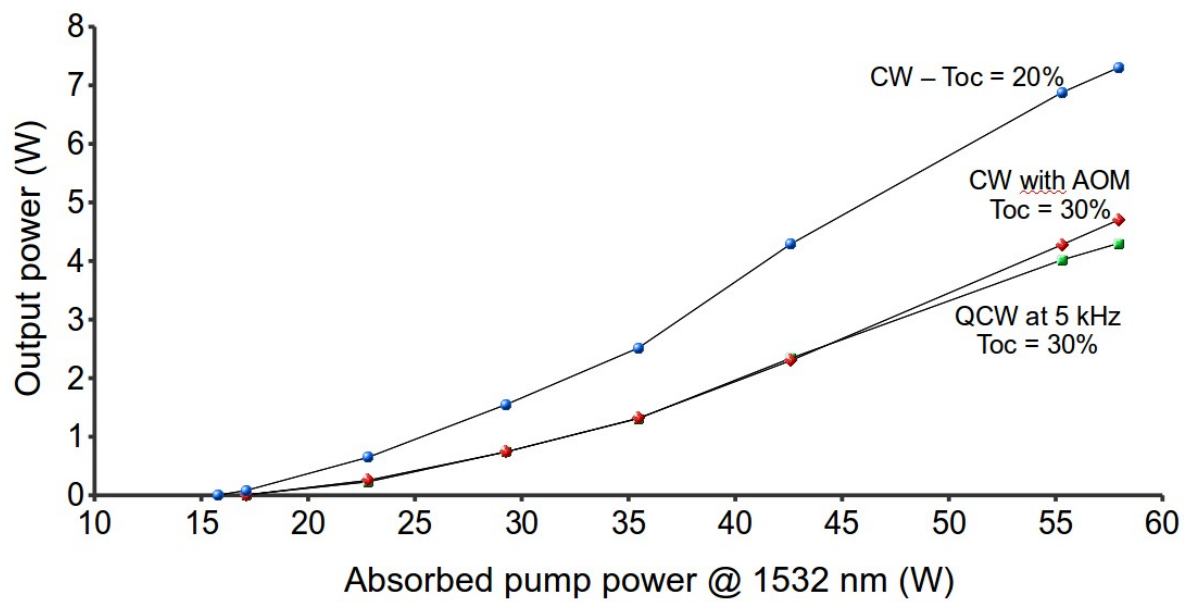

Fig. 2. Output power at $1645 \mathrm{~nm}$ versus absorbed pump power at $1532 \mathrm{~nm}$ in $\mathrm{CW}$ regime (blue cricle) with an output coupler transmission of $20 \%$. Red diamond-shape and green square are related to laser operation with the A.O.M and Toc $=30 \%$ in CW and in Q-switched operation respectively.

In order to realize Q-switched operation in the off-axis pumping configuration, an acousto-optic modulator is placed inside the cavity and an output coupler with a higher transmission $(\mathrm{T}=30 \% @ 1645 \mathrm{~nm})$ is used to prevent any damage on optics and coatings. The A.O.M. modulator introduces $7 \%$ losses inside the cavity. In this configuration, in $\mathrm{CW}$ operation the measured laser threshold is $17 \mathrm{~W}$ and the maximum output power at $1645 \mathrm{~nm}$ is $4.7 \mathrm{~W}$ for $58 \mathrm{~W}$ of absorbed pump power (see Fig. 2). In Q-switched regime, at the repetition rate of $5 \mathrm{kHz}$, the laser efficiency is identical as in CW regime. This indicates that there was no increase in losses due to up-conversion or amplified stimulated emission (ASE) when Q-switching at $5 \mathrm{kHz}$. The dependence of average output power and single pulse energy versus the repetition rate is shown on Fig. 3. 


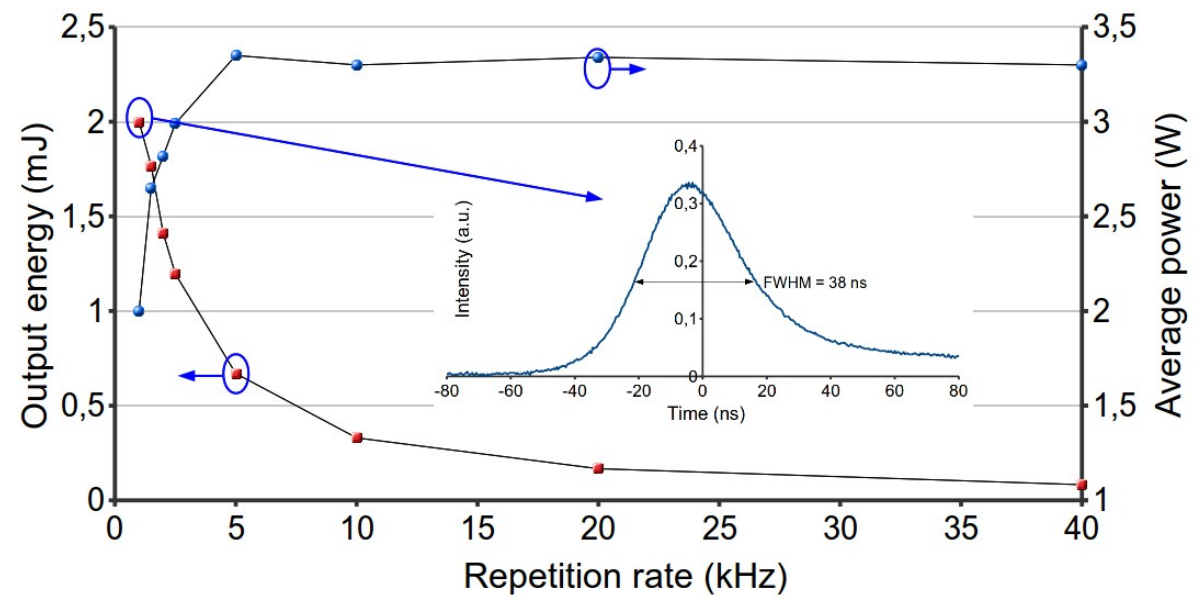

Fig. 3. Output average power (blue circle, right axis) and output single pulse energy (red square, left axis) at $1645 \mathrm{~nm}$ versus the repetition rate under $58 \mathrm{~W}$ of absorbed pump power in off-axis pumping Q-switched regime with an output coupler transmission of $30 \%$.

Inset. Temporal profile of a pulse at $1 \mathrm{kHz}$.

For the maximum absorbed pump power $(58 \mathrm{~W})$, the laser could not be operated at lower repetition rates than $1 \mathrm{kHz}$ due to damage of the optical coatings caused by the intra-cavity peak power of the pulses. At $1 \mathrm{kHz}$, the pulse energy was $2 \mathrm{~mJ}$ with a pulse duration of $38 \mathrm{~ns}$. The inset in Fig. 3 shows the temporal profile of a $2 \mathrm{~mJ}$ pulse obtained at $1 \mathrm{kHz}$. In this configuration, the pulse duration was similar to the one obtained with efficient EDFL pumping system [3] and shorter than in recent diode pumping system [12] and leads to peak-power up to $53 \mathrm{~kW}$.

\section{Conclusion}

In conclusion, we have demonstrated an efficient Er:YAG single-crystal fiber laser resonantly pumped using CW diodes at $1532 \mathrm{~nm}$. Using an off-axis pumping scheme $\mathrm{CW}$ output powers up to $7.3 \mathrm{~W}$ have been obtained and in Qswitched operation, the average power is $3,4 \mathrm{~W}$ at a $5 \mathrm{KHz}$ repetition rate. The highest single pulse energy obtained was $2 \mathrm{~mJ}$ with a pulse duration of $38 \mathrm{~ns}$ at the repetition rate of $1 \mathrm{kHz}$. To our knowledge, this is the first Q-switched directly grown Er:YAG single-crystal fiber laser. Higher pulse energy should be achieved by employing a moretransparent output coupler.

\section{Acknowledgement}

This work is supported by the DGA (Direction Générale de l'Armement) under the project FEYPIA n 200834 0019 .

\section{References}

[1] D. Chen, M. Birnbaum, P. M. Belden, T. S. Rose, and S. M. Beck, "Multiwatt continuous-wave and Q-switched Er:YAG lasers at 1645 nm: performance issues," Opt. Lett. 34, 1501 (2009).

[2] S. Setzler, M. Francis, Y. Young, J. Konves, and E. Chicklis, "Resonantly pumped eyesafe erbium lasers," IEEE J. of Quantum Electron. 11, 645 (2005).

[3] J. W. Kim, D. Y. Shen, J. K. Sahu, and W. A. Clarkson, "Fiber-Laser-Pumped Er:YAG Lasers," IEEE J. of Quantum Electron. 15, 361 (2009).

[4] Y. Young, S. Setzler, K. Snell, P. Budni, T. Pollak, and E. Chicklis, "Efficient 1645-nm Er : YAG laser," Opt. Lett. 29, 1075 (2004).

[5] N. W. Chang, D. J. Hosken, J. Munch, D. Ottaway, and P. J. Veitch, "Stable, Single Frequency Er:YAG Lasers at $1.6 \mu \mathrm{m}, "$ IEEE J. of Quantum Electron. 46, 1039 (2010).

[6] D. Garbuzov, I. Kudryashov, and M. Dubinskii, "110 W(0.9 J) pulsed power from resonantly diode-laser-pumped 1.6- $\mu \mathrm{m}$ Er:YAG laser," App. Phy. Lett. 87, 121101 (2005).

[7] I. Kudryashov, N. Ter-Gabrielyan, and M. Dubinskii, "Resonantly diode-pumped Er:YAG laser: 1470-nm versus 1530-nm CW pumping case," in Laser Technology For Defense And Security V (2009).

[8] M. Eichhorn, "High-power resonantly diode-pumped CW Er3+:YAG laser," App. Phy. B 93, 773 (2008).

[9] S. Bigotta and M. Eichhorn, "Q-switched resonantly diode-pumped Er3+:YAG laser with fiberlike geometry," Opt. Lett. 35, 2970 (2010).

[10] J. Ricard and C. Excoffon, "Patent C30B 1508; C30B 1510," (1975).

[11] I. Martial, S. Bigotta, M. Eichhorn, C. Kieleck, J. Didierjean, N. Aubry, R. Peretti, F. Balembois, and P. Georges, "Er:YAG fiber-shaped laser crystals (single crystal fibers) grown by micro-pulling down: Characterization and laser operation," Opt. Mat. 32, 1251 (2010).

[12] N.W.H. Chang, N. Simakov, D.J. Hosken, J. Munch, D.J. Ottaway and P.J. Veitch, "Resonantly diode-pumped continuous-wave and Qswitched Er:YAG laser at 1645 nm," Opt. Expr. 18, 13673 (2010). 